\title{
Mehr Chancen als Kosten
}

\section{Die Einführung der europäischen Chemikalienverordnung REACH ist umstritten. Unternehmen laufen wegen angeblich untragbarerer Kosten Sturm. Kommissi- onsvertreter und Umweltschützer weisen auf den verbesserten Umwelt- und Gesundheitsschutz durch die Verordnung hin und veranschlagen zusätzlich die Kosten wesentlich niedriger. Eine neue Untersuchung zeigt, unter welchen Be- dingugnen Unternehmen von REACH profitieren können.}

$\mathrm{D}$

Von Andreas Abrens ie EU Kommission schätzt die Kosten für die Einführung des REACH-Systems auf Ebene der Stoffhersteller auf 2,3 Milliarden Euro in elf Jahren. Zusätzlich werden über einen Zeitraum von 15 Jahren zwischen 2,8 und 5,2 Milliarden an Folgekosten für die Stoffanwender entstehen. Diese Folgekosten werden durch Veränderungen in der Verfïgbarkeit und den Preisen von Stoffen auf dem Markt während der Registrierungsphase hervorgerufen. Die Anpassungsleistung der Stoffanwender (Hersteller von Zubereitungen und Anwender) besteht im Wesentlichen darin, Stoffe zu substituieren, die aufgrund von Risiken oder aufgrund mangelnder Profitabilität nicht oder nur für beschränkte Anwendungen registriert werden. Die Schätzungen darüber, wie viele Stoffe unter REACH-Bedingungen vom Markt genommen werden, gehen weit auseinander. Die Industrie rechnet mit 20 bis 40 Prozent, die EU-Kommission mit einem bis zwei Prozent. Andere Schätzungen liegen im Bereich von drei bis 16 Prozent, je nach Marktvolumen (1). Im Verhältnis zum europäischen Chemieproduktionswert von jährlich etwa 556 Milliarden Euro mit Pharmaindustrie erscheinen die Kosten von REACH für die Chemiebranche klein. Selbst wenn man eine durchschnittliche Gewinnmarge von unter 10 Prozent ansetzt, bleiben die Kosten von REACH unter einem Prozent des jährlichen Gewinnes. Wo also liegt, wenn überhaupt, das ökonomische Problem von REACH?

\section{- Empirische Untersuchung}

Dieser Frage und auch der Frage nach dem potentiellen Nutzen von REACH gingen das Fraunhofer Institut für Systemtechnik und Innovation und das Ökopol-Institut im Rahmen einer kürzlich abgeschlossenen Studie für das Umweltbundesamt nach (2). Darin wurden unter anderem auch die Kosten und der Nutzen der neuen Chemikalienpolitik auf Unternehmensebene am Beispiel ausgewählter Wertschöpfungsketten analysiert. Besonderes Augenmerk lag auf der Balance zwischen durch REACH erzeugtem Anpassungsdruck und der Anpassungsfähigkeit der Unternehmen (vergleiche Abbildung 1). und organische Pigmente), sechs Hersteller von Farben für industrielle Anwendungen, sechs Anwender von Farben und Lacken (fünf industrielle und ein gewerblicher Anwender).

Um die zusätzlichen Effekte durch REACH herauszuarbeiten, wurden bei den Interviews die derzeit schon laufenden Entwicklungen erfragt, wie zum Beispiel die „normale“ Substitutionsquote von Stoffen.

\section{Wertschöpfungskette}

Wichtig für das Verständnis der REACH Diskussion sind die Strukturen der Chemie-Wertschöpfungskette: Die chemische Industrie in Europa besteht aus etwa 23.000 Unternehmen, 95 Prozent davon mit weniger als 250 Mitarbeitern. In diesen kleinen und mittleren Unternehmen (KMU) werden etwa 30 Prozent des Produktionswertes von etwa 36

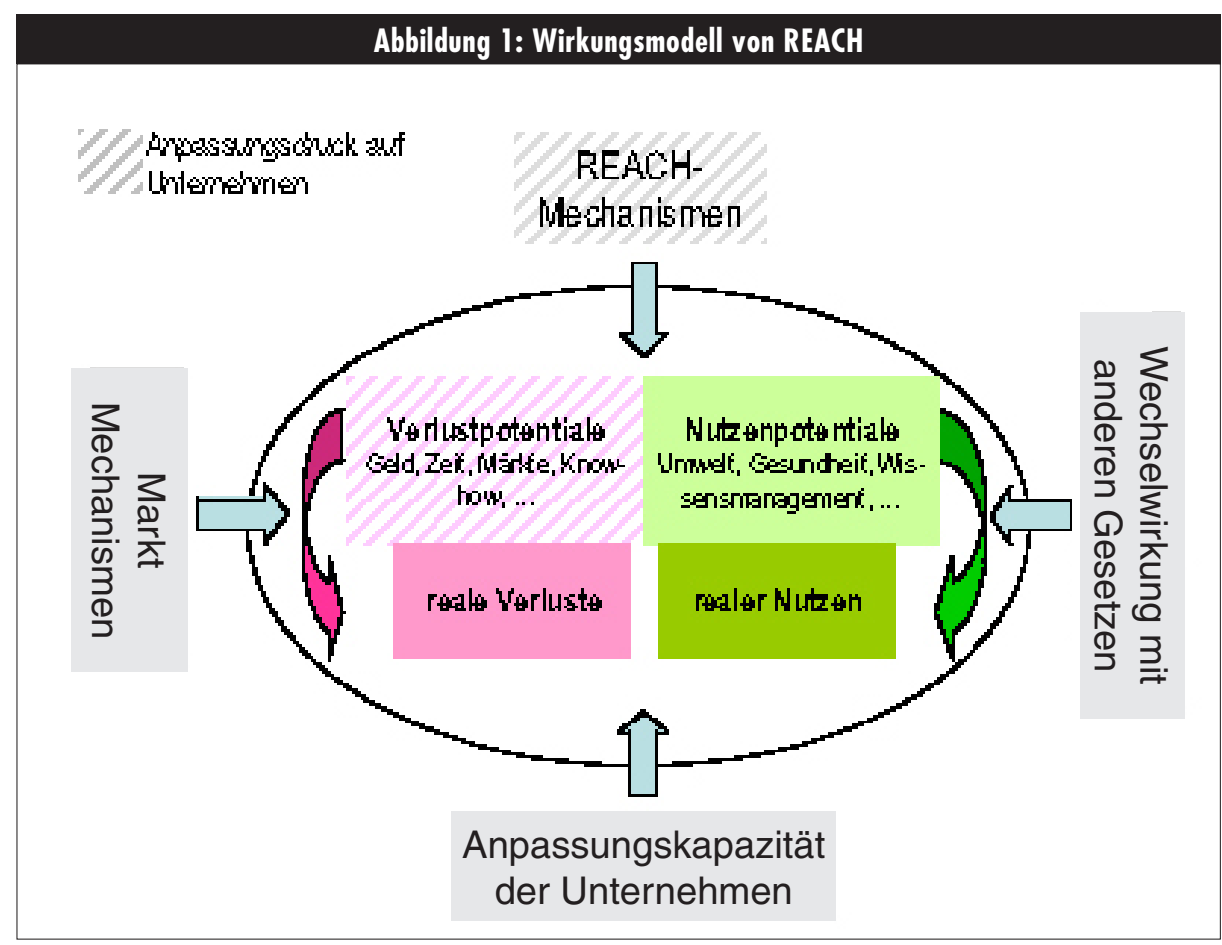

Quelle: Eigene Darstellung

Im Rahmen der Untersuchung wurden 24 Firmen auf allen Ebenen der Lieferketten interviewt, wobei der Schwerpunkt bei kleinen und mittelständischen Herstellern von Zubereitungen lag. Durchgeführt wurden

- Untersuchungen zur Wasch- und Reinigungsmittelkette: ein Importeur, ein Hersteller von Tensiden, sieben Hersteller von Zubereitungen (zwei davon für private Haushalte), vier Anwender von Zubereitungen (drei industrielle und ein gewerblicher Anwender),

- Untersuchungen zur Lackkette: ein Importeur von Additiven, zwei Hersteller von Stoffen (Additive
Prozent der Chemiebeschäftigten erzeugt. Die Arbeitsproduktivität der KMUs ist im Chemiesektor nicht kleiner als die der großen Chemiefirmen, das heißt, diese Firmen erzielen durch spezifisches Know-how in der Synthese und/oder der Kombination von Stoffen zu chemischen Produkten eine hohe Wertschöpfung.

Grundlage dafür ist einigen Branchen, zum Beispiel Herstellung von Lacken oder Herstellung von Textilhilfsmitteln, ein „,chemisches Repertoire" von mehreren hundert bis über tausend chemischen Einzelstoffen pro Betrieb und eine entsprechend hohen Anzahl von kun- 
denspezifischen Zubereitungen. Hier konzentrieren sich REACH-Kosten bei relativen kleinen Unternehmen.

\section{- Besseres Wissensmanagement}

Viele REACH-Anforderungen stehen auch schon im jetzigen Gefahrstoffrecht. REACH schafft allerdings die Anreize und firmenübergreifenden Kommunikationsmechanismen, um deren Umsetzung zu verbessern. REACH schafft mit der Stoffsicherheitsbewertung und den neuen Pflichten der Stoffanwender ein System, in dem die bereits vorhandenen, aber in der Wertschöpfungsketten verteilten Informationen in iterativen Prozesses zusammengeführt und ausgewertet werden.

Neue Tests oder Expositionsmessungen werden nur da notwendig, wo geeignete Daten tatsächlich fehlen. Der Stoffhersteller ermittelt die Eigenschaften seines Produktes und definiert die sicheren Anwendungsbedingungen (Expositionsszenario) für die ihm bekannten Anwendungen. Diese werden über das Sicherheitsdadern als praktische Hilfe.

Falls der Stoffanwender diesen Vorgaben des Herstellers nicht folgen kann oder will, muss er entweder mit diesem in Kontakt treten oder selbst die Verantwortung übernehmen (vergleiche Abbildung 2). Diese Mechanismen sind auch in der gegenwärtigen Sicherheitsdatenblattrichtlinie, Technische Regeln Gefahrstoffe 220, angelegt. Im Rahmen des REACH Systems wird tenblatt mitgeteilt und dienen den Stoffanwen-

aber die Motivation zur Umsetzung vergrößert, da daran die Vermarktungsrechte für den Stoffhersteller hängen und die Verfügbarkeit von Rohstoffen für den Stoffanwender.

\section{Mehr Wettbewerb um Sicherheit}

Gegenwärtig wissen die Hersteller von Lacken, Reinigungsmitteln und anderen Zubereitungen für industrielle Kunden nicht, in welchem Umfang sie unzureichend geprüfte Stoffe von ihren Vorlieferanten bekommen. Nur wenige Lackhersteller haben beispielsweise ein einzelstoffbezogene Inventar ihrer Rohstoffe mit den entsprechenden Stoffidentitäten und der Möglichkeit, systematisch Informationslücken zu erkennen. Mehr noch, gegenwärtig ist es noch immer ein Wettbewerbsvorteil, wenn ein Stoff aus Datenmangel nicht als gefährlich gekennzeichnet werden muss.

Diese Wettbewerbsverzerrung wird durch REACH behoben, Stoffe können auf vergleichbarer Informationsgrundlage beurteilt werden und die Stoffanwender können einen für ihre Zwecke sicheren Stoff auswählen. REACH beseitigt insofern ein wichtiges Wettbewerbshindernis. Allerdings stellte sich in der Studie auch heraus, dass kaum ein Unternehmen damit rechnet, von seinen Kunden einen höheren Preis für einen „REACH- dokumentierten" Stoff zu erhalten.

Normale mittelständische Hersteller von Industrielacken handhaben zwischen 400 und 1400 Rohstoffen. Wenn sie diese Stoffe an ihre Kunden weitergeben, übernehmen sie Produktverantwortung

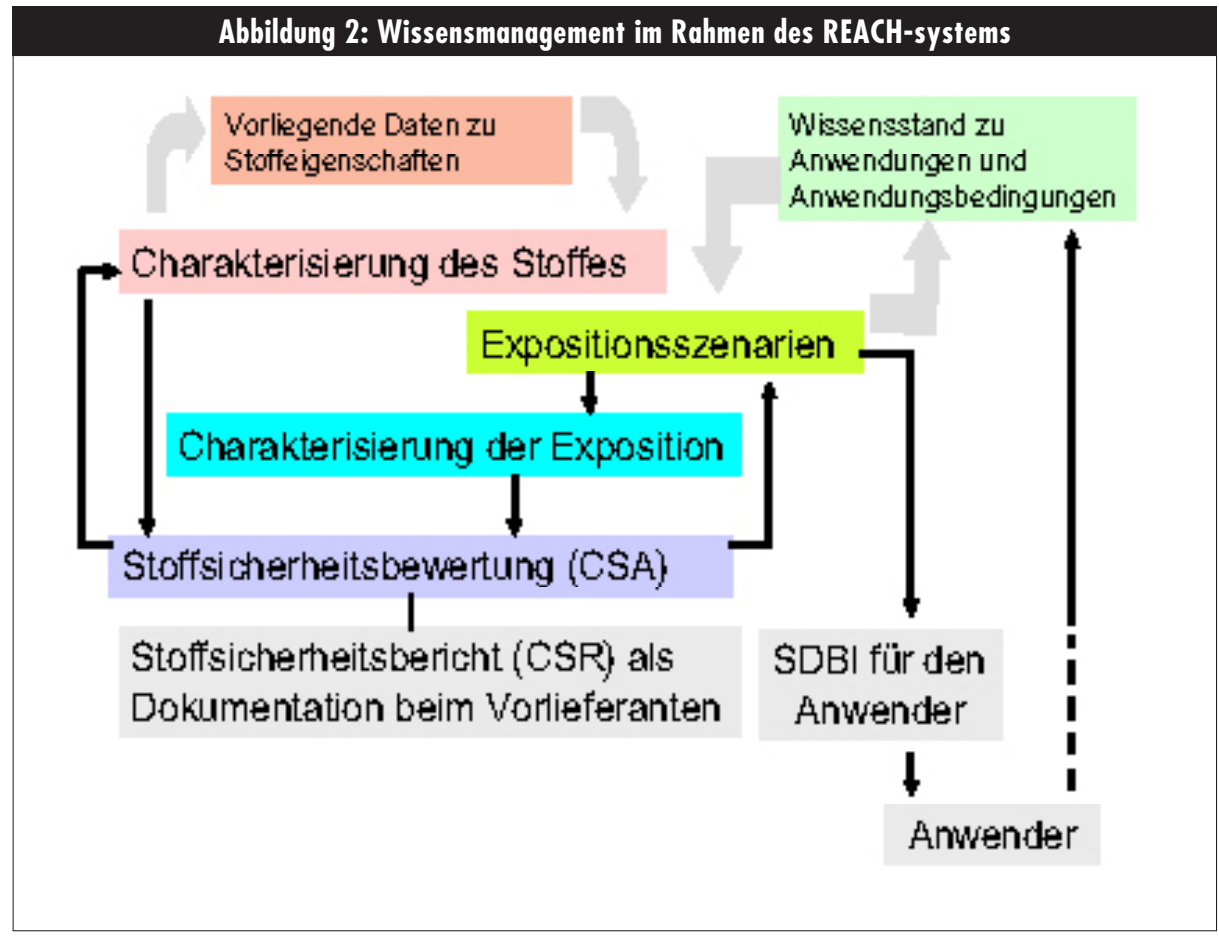

Quelle: Eigene Darstellung in einem kaum kalkulierbaren Ausmaß. Umgekehrt wissen Stoffhersteller häufig nicht, unter welchen Bedingungen und für welche Zwecke ihre Produkte im Markt eingesetzt werden und bekommen von den Anwendern auch keine Information darüber. REACH wird hier eine Grundlage für mehr Transparenz und Verantwortungszuordnung schaffen, die auch für Produkthaftungsfragen und globale Product Stewardship notwendig ist.

\section{Verringerung der Stoffvielfalt?}

Die Kosten der REACH-Registrierung können unter bestimmten Bedingungen dazu führen, dass es für den Hersteller unwirtschaftlich erscheint, den Stoff zu registrieren. Für Tenside in industriellen Wasch- und Reinigungsmitteln, für Lack-Additive und für organische Pigmente wurden im Rahmen der Studie Marktpreise und spezifische Kosten der Registrierung miteinander verglichen:

- Marktpreise bei Tensiden (< 100 Tonnen pro Jahr) etwa ein bis drei Euro pro Kilogramm. Marktpreise bei Lackadditiven und Pigmenten (< 100 Tonnen pro Jahr) etwa fünf bis 23 Euro pro Kilogramm

- Auf das Produktionsvolumen eines Jahres umgelegt, betragen die durchschnittlichen Registrierkosten in diesem Tonnageband (basierend auf Schätzungen des Europäischen Chemikalienbüros) zwischen 0,5 und 16 Euro pro Kilogramm.

Das heißt, für den Hersteller können sich, unter der Annahme, dass eine Kostenüberwälzung nicht möglich ist, sehr lange pay-back Zeiten ergeben. Er würde den Stoff vom Markt nehmen. Allerdings heißt das nicht, dass der Stoff ganz vom Markt verschwindet, möglicherweise tritt nur eine Konzentration bei anderen Herstellern ein. Und selbst wenn der Stoff nicht mehr auf dem Markt verfügbar wäre, kann die nachgefragte Funktionalität möglicherweise trotzdem über andere Stoffe abgedeckt werden (Substitute). So rechnet zum Beispiel der befragte Tensidhersteller damit, dass er 40 Prozent seiner Stoffe aus dem Portfolio nehmen würde, das aber nur bei fünf bis zehn Prozent der Stoffe tatsächlich zum Verschwinden vom Markt führen würde.

Die Substitutionselastizität zwischen den einzelnen Stoffen ist im Bereich der Spezialitäten wie zum Beispiel bei Lackadditiven kaum zu quantifizieren, da es sich hier um das unmittelbare Knowhow der Lackhersteller handelt. Teilweise für die industriellen Kunden „Einzelanfertigungen“ von Lacken hergestellt. 


\section{Das REACH-System im Überblick}

Am 29. Oktober 2003 hat die EU Kommission ihren Vorschlag für eine Europäische Verordnung über die Registrierung, Bewertung, Zulassung und Beschränkung von Chemikalien (REACH) vorgelegt. Die Registrierung und die damit verbundene Kommunikation zwischen Stoffherstellern und Stoffanwendern stellen einen Systemwechsel dar:

- Etwa 30.000 Altstoffe müssen nach einem verbindlichen Zeitplan innerhalb von 11 Jahren bei einer zentralen EU-Behörde mit einem definierten Satz von Informationen registriert werden. Nicht registrierte Stoffe dürfen nach Ablauf der Fristen nicht mehr produziert oder verwendet werden.

- Die Hersteller und Importeure von Stoffen mit einem Marktvolumen (pro Hersteller) von mehr als 10 Tonnen (etwa 11.000 Stoffe) müssen eine Chemikaliensicherheitsbewertung (CSA) durchführen. Ist der Stoff gefährlich, ist zusätzlich eine Expositionsbewertung und eine Risikocharakterisierung für alle Stufen des Stoff-Lebenszyklus gefordert. Das heißt, für die Registrierung muss der Stoffhersteller die Anwendungen seines Produktes im Markt und die Anwendungsbedingungen durchdenken, Expositionen abschätzen und geeignete Maßnahmen zum Risikomanagement definieren. Dazu gehört auch, sich auf bestimmte Anwendungsbereiche festzulegen (zum Beispiel: „Keine direkte oder indirekte Anwendung in Verbraucherprodukten") und es nicht allein dem Markt zu überlassen, wer welchen Stoff für was einsetzt.

- Weicht der Hersteller einer Zubereitung, wie zum Beispiel eines Lackes, von diesen Vorgaben ab, geht die Pflicht zur Sicherheitsbewertung auf ihn über. Das Gleiche gilt für die industriellen Anwender von Farben, Lacken, Schmierstoffen oder Textilhilfsmitteln.

- Um Tierversuche einzusparen und den Aufwand für die Erzeugung neuer Daten zu begrenzen, enthält der Verordnungsentwurf verschiedene Mechanismen zur Nutzung bereits vorhandener Datenbestände.
Die Große Spanne der möglichen Registrierkosten kommt dadurch zu Stande, dass die Kosten weniger von der Verordnung selbst abhängen, sondern der Art und Weise, wie es Industrie und Behörden gelingt das REACH-Konzept mit effizienten Instrumenten und Prozessen umzusetzen. Selbst wenn Stoffe vom Markt verschwinden sollten, können die Hersteller von Zubereitungen in gewissen Umfang damit umgehen. In beiden Wertschöpfungsketten wurde die „normale“, von außen erzwungene Substitutionsquote ermittelt. Diese bezieht sich auf Substitutionen, die von außen durch neue Gefahrstoffeinstufungen oder Veränderungen in der Verfügbarkeit von Roh- stoffen ausgelöst werden. Bei den befragten Firmen lag diese Quote, hochgerechnet auf zehn Jahre, bei 5 bis 20 Prozent des jeweiligen Rohstoffportfolios. Das heißt, Stoffaustausch ist ein normaler Vorgang und gerade auch für innovative Unternehmen nichts ungewöhnliches. Die Unternehmen können sich an mögliche REACH-Effekte anpassen (vergleiche Abbildung 3)

Allerdings kostet die Reformulierung von Zubereitungen Innovationsressourcen, die für die Zeit der REACH Umsetzung an andere Stelle fehlen. Deshalb sollten die Kosten der Registrierung und damit die Wahrscheinlichkeit des Stoff-Entfalls möglichst eng mit den Umwelt- und Ge-
Abbildung 3: Anpassungsmechanismen an REACH innerhalb der Produktionskette

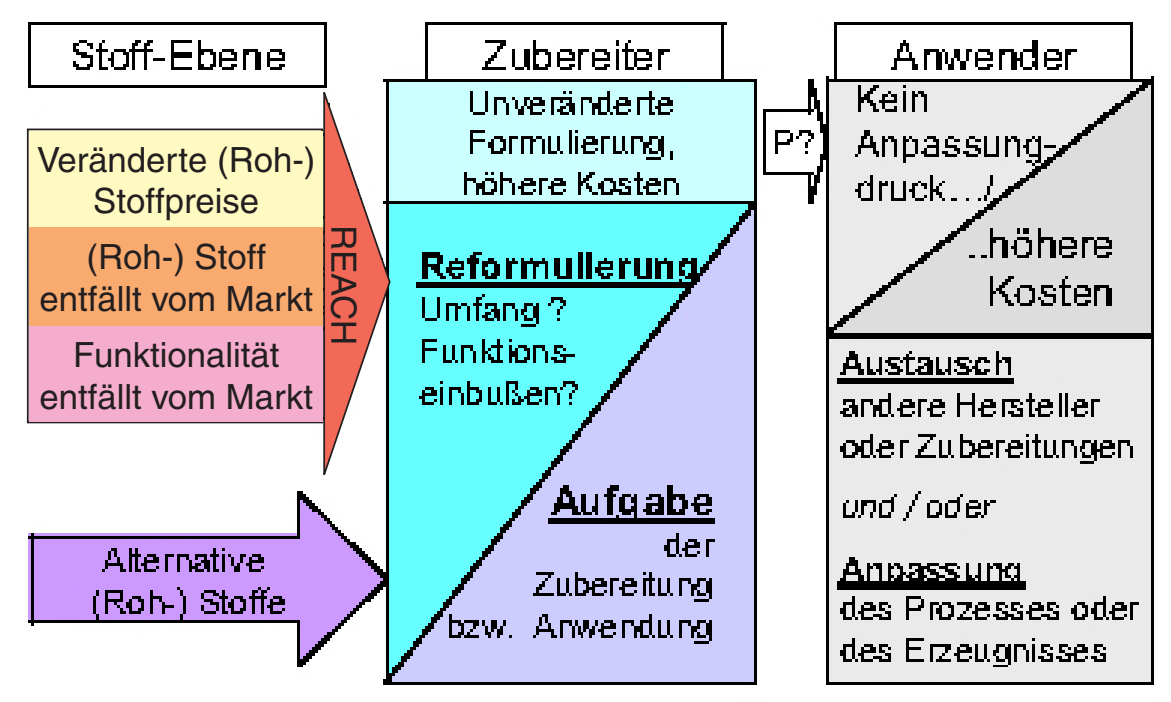

Quelle: Eigene Darstellung sundheitsrisiken korrelieren, damit REACH schon bei der Stoffregistrierung ein Erfolg wird: Stoffe sollten dort entfallen, wo potentielle Risiken die Registrierung teuer machen und nicht einfach nur, weil ein kleines Marktvolumen und teure Standardtests zu hohen spezifischen Registrierkosten führen. Hier besteht in den nächsten Jahren noch ein erheblicher Entwicklungs- und Abstimmungsbedarf für die einzelnen Elemente des REACH-Systems.

\section{Kooperation notwendig}

Zudem wird der Erfolg von REACH davon abhängen, inwieweit Industrie und Behörden der Rollenwechsel gelingt und in gemeinsamen Projekten genug Vertrauen aufgebaut wird, um Verantwortung an die Industrie zu übertragen und REACH als flexibles System zum Wissensmanagement und zur Lokalisierung von Produktverantwortung zu nutzen.

\section{Anmerkungen}

(1) Risk and Policy Analysts Limited (Hrsg.): Availability of low value products and product rationalisation - Assessment of the business impacts of new regulations in the chemicals sector - Phase 2. Loddon 2003.

(2) Ostertag, K.,/ Marscheider-Weidemann, F./ Angerer, G./ Ahrens, A.,/ Meyer, U.: Analyse der Kosten und Nutzen der neuen EU-Chemikalienpolitik - Untersuchung anhand ausgewählter Branchen unter Beachtung der Wirkungen auf Wettbewerbsfähigkeit, Innovation, Umwelt und Gesundheit. Hamburg 2004.

\section{Der Autor}

Andreas Ahrens ist Mitarbeiter bei Ökopol-Institut für Ökologie und Politik.

Kontakt: Ökopol, Nernstweg 32 -34, 22765 Hamburg. Tel.040-3910020,E-Mail: info@oekopol.de 
(c) 20I0 Authors; licensee IÖW and oekom verlag. This is an article distributed under the terms of the Creative Commons Attribution Non-Commercial No Derivates License (http://creativecommons.org/licenses/by-nc-nd/3.o/), which permits unrestricted use, distribution, and reproduction in any medium, provided the original work is properly cited. 